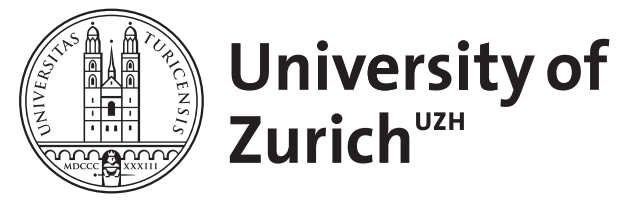

Zurich Open Repository and Archive

University of Zurich

University Library

Strickhofstrasse 39

CH-8057 Zurich

www.zora.uzh.ch

Year: 2010

Kommentar I zum Fall "Behandlungsabbruch bei Anorexie?"

Wild, V; Krones, T

DOI: https://doi.org/10.1007/s00481-010-0058-z

Posted at the Zurich Open Repository and Archive, University of Zurich

ZORA URL: https://doi.org/10.5167/uzh-39845

Journal Article

Published Version

Originally published at:

Wild, V; Krones, T (2010). Kommentar I zum Fall "Behandlungsabbruch bei Anorexie?". Ethik in der Medizin, 22(2):133-134.

DOI: https://doi.org/10.1007/s00481-010-0058-z 


\title{
Kommentar I zum Fall: „Behandlungsabbruch bei Anorexie?“
}

\author{
Verina Wild · Tanja Krones
}

Online publiziert: 31 . März 2010

(C) Springer-Verlag 2010

Der Fall der jungen Patientin mit Anorexia nervosa demonstriert in eindrucksvoller Weise, was es bedeutet, einem moralischen Dilemma gegenüberzustehen.

Das Fürsorgeprinzip scheint nahezulegen, die Patientin - ggf. auch unter Sedierung zwangszuernähren. Die Anorexia nervosa weist insgesamt eine hohe Mortalität auf. Der BMI der Patientin ist extrem niedrig, vielfache Therapieversuche sind fehlgeschlagen. Die Wahrscheinlichkeit, dass die Patientin mittelfristig an ihrer Erkrankung stirbt, ist sicher als hoch einzuschätzen, wobei gemäß der Schilderung des Falles nicht unbedingt davon auszugehen ist, dass eine akute Gefahr für das Leben der Patientin besteht.

Die Achtung vor der Autonomie der Patientin, die sich mehrfach dafür ausspricht, ,ihre Ruhe haben“" zu wollen, und daher ,,palliativ“ behandelt zu werden, spricht gegen die Fortführung einer Behandlung, die auf Normalisierung des Essverhaltens und Gewichtszunahme zielt.

Auch das Prinzip der Gerechtigkeit im Sinne einer Weiterbehandlung im Universitätsklinikum ist für die Frage, welcher therapeutische Weg eingeschlagen wird, von Bedeutung. Die Patientin bindet viele Ressourcen des Akutkrankenhauses, die anderen Patienten fehlen.

Nach zweimonatiger Behandlung zeigt sich kein richtungsweisender Behandlungserfolg, sondern eher eine Verschlechterung der Situation, so dass eine Verlegung der Patientin angedacht wird. Nur - wohin? Die Aufnahme in das Universitätsklinikum schien bereits eine Maßnahme ,of last resort“ gewesen zu sein.

Die spezifische Fallkonstellation ist zudem mit einer hohen emotionalen Betroffenheit der Beteiligten und mit komplexen Beziehungskonstellationen verbunden, die auch vom Leser erspürt werden können: die Verzweiflung der Eltern, die emotionalen Konflikte im

\footnotetext{
V. Wild $(\bowtie)$

Institut für Biomedizinische Ethik, Universität Zürich,

Pestalozzistr. 24, 8032 Zürich, Schweiz

E-Mail: wild@ethik.uzh.ch

T. Krones

Klinische Ethik, Universitätsspital und Universität Zürich, Zürich, Schweiz
} 
Behandlungsteam, die extreme Belastung der Therapie für die Patientin. Alle Beteiligten kreisen um das Leben der Patientin, greifen ein, misstrauen sich teilweise gegenseitig - der Vater droht bereits mit juristischen Schritten - während die Patientin nur noch eines will: „in Ruhe gelassen zu werden“.

Ein Ausweg aus der Situation erscheint schwierig. Im Rahmen einer prinzipienethischen Abwägung wäre zu überlegen, wie die Konflikte zwischen den Prinzipien so abgewogen werden können, dass deren jeweilige Bedeutung in dem Fall genauer spezifiziert wird. Ziel wäre ein Konsens bezüglich einer bestmöglichen Realisierung der Prinzipien, der von der Patientin mitgetragen werden kann.

Bezüglich der Spezifikation des Prinzips der Autonomie ergibt sich die Schwierigkeit, wie dieses bei einer schwer anorektischen Patientin mit Depressionen und Zwangsstörungen $\mathrm{zu}$ verstehen ist. Die Literatur kommt hier zu keinem einheitlichen Schluss. Einige gehen davon aus, dass die freie Willensbildung aufgrund der Angst der Patientinnen vor dem Essen und dem von der Norm abweichenden Körperschema eingeschränkt ist. Die Ablehnung der - potentiell heilenden - Behandlung sei also am ehesten als ein Symptom der Erkrankung einzuschätzen, eine Zwangsbehandlung sei daher in Einzelfällen geboten.

Doch auch psychosomatisch-psychiatrische Erkrankungen wie die Anorexia nervosa können unheilbar und mit einer dauerhaft schlechten Lebensqualität verbunden sein. Verläuft trotz vieler Behandlungsversuche die Erkrankung chronisch, mehrfache Therapiebemühungen schlagen fehl, die Lebensqualität ist zunehmend schlecht, und die Patientinnen äußern sich immer wieder dahingehend, lebensverlängernde, potentiell kurative Therapien nicht mehr weiter fortführen zu wollen, so kann dies auch als eine wohlabgewogene Entscheidung im Hinblick auf eine Therapiezieländerung gelten. Eine solche Einschätzung liegt durch die behandelnden Psychiater in dem geschilderten Fall vor. Die Patientin wird dabei nicht als suizidal eingeschätzt, es ist nicht ihr Wunsch zu sterben, sondern ihr Ziel ist es ,,in Ruhe gelassen zu werden“, sie nimmt die Möglichkeit zu sterben jedoch als Nebenfolge in Kauf.

Eine Zwangsernährung ist in dem vorliegenden Fall nicht ohne Risiko. Die Behandlung muss zudem im Sinne der Freiheitsrechte verhältnismäßig sein, kann daher nur temporär und zur Abwehr einer unmittelbaren Gefahr eingesetzt werden. Der massive Eingriff in die Autonomie der Patientin könnte zudem dazu führen, dass die therapeutische Vertrauensbasis zerstört wird. Dabei könnte die mittelfristige Prognose schlechter werden als bei einer symptomorientierten Behandlung, die der Patientin zumindest vorübergehend überlässt, wann sie wie viel essen möchte. Hierdurch könnte auch der Druck, den die Patientin gemäß der Fallschilderung durch die langjährige Krankheitssituation und die engmaschige Fürsorge, immer wieder gepaart mit Zwangsbehandlungen, erlebt hat, sinken und bestenfalls ein Freiraum entstehen, welcher eigene Entscheidungen zum Leben erst wieder ermöglicht.

Ein aus unserer Sicht bestmöglich tragbares Konzept wäre es, die Patientin auf ihren Wunsch in eine Einrichtung zu verlegen, in welcher sowohl eine längerfristige palliative wie potentiell kurative Behandlung möglich wäre. Der Patientin sollten dort weiterhin verschiedene Behandlungsangebote (medizinisch, psycho-/familientherapeutisch) gemacht werden. Die Eltern sollten soweit mit einbezogen oder ausgeschlossen werden, wie dies von der Patientin selbst toleriert bzw. gewünscht wird und zu einem Therapieerfolg beitragen kann. Wichtig erscheint es, der Patientin deutlich zu machen, dass ihre Entscheidungen bezüglich einer auf Symptome orientierten Behandlung ernst genommen und gegebenenfalls vom Behandlungsteam mitgetragen werden, sie aber auch jederzeit revidiert werden können. 